inhibition
of tumour
growth was
observed, with
higher doses
achieving a
sustained
tumour
regression

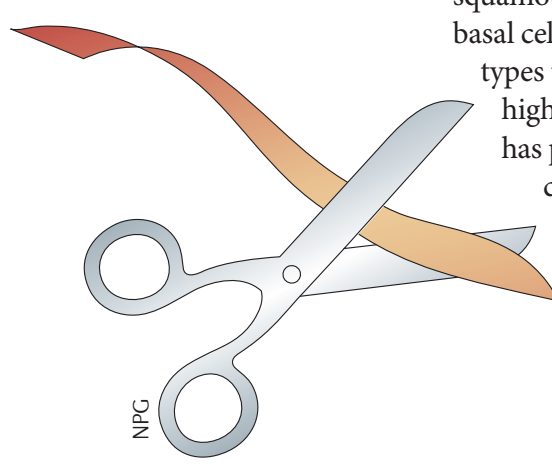

\title{
THERAPEUTICS
}

\section{Antitumour potential of catalytic DNA}

Of the emerging targeted cancer therapies, small-molecule inhibitors and antibodies receive the greatest attention. However, a new report shows that a catalytic DNA molecule (DNAzyme) that targets JUN expression shows promising in vivo efficacy for treating some types of skin cancer, and has an encouraging safety profile.

DNAzymes are synthetic singlestrand DNA molecules that can cleave RNAs. Levon Khachigian and colleagues investigated the DNAzyme Dz13, which targets transcripts of the JUN oncogene and which has been shown to inhibit tumour growth and angiogenesis in some mouse models of cancer. In the current study, the authors assessed Dz13 for the treatment of mice with established squamous cell carcinoma (SCC) or basal cell carcinoma (BCC), tumour types that are known to express high levels of JUN. Because DNA has poor tissue penetration and cell permeability, Dz13 was injected intratumorally in a liposomal formulation. A dose-dependent inhibition of tumour growth was observed, with higher doses achieving a sustained tumour regression. The antitumour effects were greater in immunocompetent mice than in immunodeficient mice, implying that an immune response contributes to the activity of Dz13. Indeed, both $\mathrm{CD}^{+}$(cytotoxic) and $\mathrm{CD}^{+}$(helper) $\mathrm{T}$ cells were found to infiltrate tumours undergoing treatment, and depletion of either cell type reduced Dz13 efficacy. However, the immunogenic targets recognized by these T cells are currently unclear.

Dz13 treatment had the expected effect of JUN downregulation in tumours. Additional molecular effects included the downregulation of cell cycle proteins, invasion-associated matrix metalloproteinases and the angiogenic marker vascular endothelial growth factor A (VEGFA), and the induction of apoptosis. One area of debate in the DNAzyme field is the contribution of off-target effects to efficacy. The authors used multiple approaches, including the use of scrambled or catalytically dead forms of Dz13, to conclude that the antitumour effects were primarily through JUN targeting.

The pharmacokinetics, safety and tolerability of Dz13 were also examined. Key findings were that intravenous or intradermal Dz13 administration to healthy rats, miniature pigs or cynomolgus monkeys caused no substantial toxicities - as shown by parameters that included body weight, liver function, and histological and biochemical analyses - even at doses that were in great excess of the efficacious antitumour doses in mice. Additionally, Dz13 did not score for unwanted binding to any of $>70$ biologically relevant targets. These safety assessments imply that human clinical trials of Dz13 are feasible, although which human cancer subtypes might be most responsive to Dz13, and whether patients should be stratified according to JUN expression levels are currently unclear.

Importantly, owing to the intratumoral delivery route used in the efficacy studies it will be challenging to achieve similar efficacies in less accessible tumours or in metastatic disease. However, the capacity to custom design DNAzymes makes them promising tools for rapidly assessing the value of targeting the many putative oncogenes that are emerging from cancer genomics studies.

Darren J. Burgess

ORIGINAL RESEARCH PAPER Cai, H. et al. DNAzyme targeting c-jun suppresses skin cancer growth. Sci. Transl Med. 4, 139ra82 (2012). 